\title{
Children's Down Behavior Syndrome: Difficulty Concentrating and Obsessive-Compulsive Disorder
}

\author{
Maria Sclanavo ${ }^{1}$, Gyula Sebes ${ }^{1}$ \\ ${ }^{1}$ Semmelweis University, Hungary
}

\begin{abstract}
This article discusses the behavior of children with Down syndrome who have difficulty concentrating and obsessive-compulsivity, characteristics of Down syndrome children, factors that cause Down syndrome children, problems with Down syndrome sufferers and cognitive and behavioral problems. The development of a child with Down Syndrome is certainly different from the development of a healthy child. Excessive expression of chromosomes leads to a decrease in the number of nerves in the central nervous system, delayed myelination, disruption of cell cycle regulation, and causes excessive protein production and abnormal neurotransmission. Problems for people with Down syndrome are hearing problems and impaired vision, congenital heart disease, and growth problems when they were babies. The tendency for psychological and mental disorders in children with Down syndrome can cause compulsive obsession. Obsession is a thought or image that cannot be prevented and continues to exist in a person's consciousness even if he views it as something unpleasant and wants to avoid. A compulsion is a stereotypical act that prompts a person to repeat the action.
\end{abstract}

Keywords: Child Behavior, Down Syndrome, Obsessive-Compulsive

Received: October 17, 2020

Revised: November 5, 2020

Accepted: November 14, 2020

\section{Introduction}

The phenomenon of Down syndrome occurs approximately one in 800 to 1,000 births of a baby (Brain Research Succee Stories, 2005). This disorder is a genetic disorder that affects more than 5,000 births of babies in the United States each year (Becky, 2006). Some people with disabilities can be identified based on their physical characteristics and some are not. One example of a person with a disability that we can recognize physically is a person with Down syndrome. We can identify Down syndrome based on the characteristics of a flat face (such as the Mongoloid race), slightly crossed eyes, small mouth, small head, short neck, and relatively short hands and feet (Evans-Martin, 2009: 14).

Down syndrome, a child with special needs, which is a condition of underdevelopment in physical and mental development in children caused by abnormalities in the development of children with mental disabilities, generally have more disorders than other disabilities, especially their intelligence. Almost all cognitive abilities of mentally handicapped children have disorders such as slow learning, ability to overcome problems, lack of ability to make causal relationships, so that their appearance is very different from other children. Mentally disabled children are characterized by weak motor control, lack of ability to coordinate, but on the other hand, they can still be trained to reach the normal point.

Other signs such as reading a book close to the eyes, the mouth is always open to understand something, meaning takes a long time, has sensory difficulties, experiences speech impediments and verbal development. 
This type of Down syndrome is a type of specificity in early childhood which is a result of excess chromosomes while in the womb (Hulten et al., 2009). Down syndrome occurs because of an abnormality of the 21st chromosome arrangement of 23 human chromosomes. In normal humans, 23 chromosomes pair up to 46. In people with Down syndrome, the number 21 chromosome is three (trisomy), bringing the total to 47 chromosomes. This excessive amount results in a shaking of the cellular metabolic system, which eventually leads to Down syndrome.

Children with Down syndrome are usually less able to coordinate between gross and fine motor skills. For example, difficulty wearing button-up clothes and putting on strappy shoes yourself. In addition, children with Down syndrome also have difficulty coordinating cognitive abilities and language, such as understanding the benefits of an object. It is difficult for children with Down syndrome to understand the functions and uses of objects around them.

In general, the average IQ of children with Down syndrome is 50 ( Pitzutillo \& Herman, 2009). This happens from infancy until the next process. The development of IQ at the age of 16 to 40 weeks is around 71-75, at the age of one year 69 and at the age of 18 months to 58 . Patients with Down syndrome may experience decreased sensorimotor development in competence and a low level of maximal growth (Sullivan, 2003). People with Down syndrome are indicated to have a less efficient Information Processing Model such as visual development than normal development as a whole. This may be related to the barrier to maturity in the visual cortex (Fang, 2019).

\section{Understanding Down Syndrome}

According to Fisch et al. (2003) Down syndrome is not a disease but a chromosomal disorder that can occur in both men and women, and this chromosomal abnormality is not always passed on to the next offspring. Most of the chromosomal abnormalities in trisomy 21 cases occurred $94 \%$ due to chromosome additions, $3 \%$ due to translocation, $2 \%$ mosaic type and the remaining $1 \%$ consisting of rare chromosomal abnormalities.

Down syndrome is a condition of underdeveloped physical and mental development of children due to abnormalities in chromosomal development. Chromosomes are special fibers that are present in every cell in the human body, where there is genetic material that determines a person's characteristics. Down syndrome occurs because of an abnormality of the 21st chromosome arrangement of 23 human chromosomes. In normal humans, 23 chromosomes pair up to 46. In people with Down syndrome, the number 21 chromosome is three (trisomy), bringing the total to 47 chromosomes. This excessive amount results in a shaking of the cellular metabolic system, which eventually leads to Down syndrome.

There are several types of dow syndrome namely trisomy 21 , translocation and mosaic. Trisomy 21 is a common disorder where there are 3 chromosomes 21 ( Antonarakis et al., 2004). Trisomy is caused by meiosis or the failure of the chromosome pairs to separate (27). 95\% of people with Down's Syndrome are caused by trisomy 21.

\section{Characteristics of Children with Down Syndrome}

Patients with characteristic signs are very easily recognized by the presence of a prominent physical appearance in the form of a head that is relatively small than normal (microchephaly) with a horizontal anteroposterior part of the head. On the face there is usually a flat nose, a smaller mouth and a protruding tongue (macroglossia). Often the eyes are narrowed with the center corner forming folds (epicanthal folds). Clinical signs on other parts of the body are short hands including the knuckles and the distance between the first 
and second fingers on both hands and feet is wide. The relatively short stature, the shrunken head, the flat nose resembling a Mongolian, it is also known as the Mongoloid. Meanwhile, the skin layer usually appears wrinkled (dermatoglyphics).

Children with Down syndrome suffer from various deficits in learning and development. They tend to be uncoordinated and lack sufficient muscle pressure making it difficult for them to perform physical tasks and engage in play activities like other children. These children have memory deficits, especially for information displayed verbally, making it difficult to learn at school. They also have difficulty following teacher instructions and verbally expressing their thoughts or needs clearly. Despite these difficulties, most can learn to read, write, and perform simple arithmetic tasks if they receive proper education and good support (Iverson \& Braddock, 2011)

Some of the symptoms that arise due to Down syndrome. Abbasi Moheb (2012) stated that these symptoms can vary from being completely invisible, seemingly minimal, to the following observable features: Physical appearance appears through the head that is relatively smaller than normal (microchepaly) with the anteroposterior part of the head horizontal. A face similar to that of a Mongol, between a flat nose, the base of the kemek nose. The distance between the two eyes is far away and the skin over the inner corner. The size of the mouth is small, but the size of the tongue is large and causes the tongue to always stick out (macroglossia). The dentition of people with Down syndrome is slow and irregular. Lower ear level and slightly short neck. Often the eyes are narrowed with the center corner forming $80 \%$ epicanthol folds. People with Down syndrome experience chewing, swallowing and speaking disorders. Hypogenitalism (penis, scrotum, and small testes), hypospadias, cryptorchism, and delayed development of puberty. People with Down syndrome have soft, dry, and thin skin. Meanwhile, the skin layers usually appear wrinkled (dermatologlyphics). The hands are short, the knuckles of the fingers and the distance between the first and second fingers are short, both the hands and the feet are wide. They also have short fingers and a little finger bent inward. The palms of their hands usually only have one vein called "simian crease". The feet are rather short and the distance between the mother's feet and the second toes is a bit far apart. The muscles are so weak that they become flaccid and face problems with gross motor development. Problems related to problems such as failure of internal organs, especially the heart and intestines. The small bones in the neck are unstable, causing paralysis (atlantaoxial instability). A small proportion of sufferers have the potential to develop white blood cell cancer or leukemia. The learning development problem of people with Down syndrome as a whole has developmental retardation and weakness of reason. In the early stages of development, they experience slow problems in all aspects of development, namely slow walking, fine motor development and speech.

The IQ of people with Down syndrome is below 50. By the time they are 30 years old, they may develop dementia (memory loss, decreased intelligence, and personality changes) (Lautarescu et al., 2017)

\section{Factors Causing Children with Down Syndrome}

For mothers aged 35 years and over, during pregnancy, they have a higher risk of giving birth to Down Syndrome children. Ninety-five people with Down syndrome are caused by excess chromosome 21. This condition is caused by the "non-dysjunction" of the chromosome involved, namely chromosome 21 , which during the process of mitotic cell division, the separation of chromosome 21 does not work completely.

Down syndrome occurs not due to external factors, Down syndrome occurs due to chromosomal deficiencies as a result of a genetic accident which can be detected through 
amniosynthetic testing. Doctors emphasize that Down syndrome is not linked to everything parents do either before or during pregnancy. Down syndrome occurs not because of the food or drink that the mother consumes when pregnant, nor traumatic feelings, nor does the mother and father commit or regret what they have experienced.

Down syndrome is caused by the presence of an extra chromosome in every cell of the body, another factor that causes a high risk of having Down syndrome children is old age. The older the mother, the more the mother has the opportunity to give birth to Down syndrome children. An increase in the chances of giving birth to a Down syndrome child occurs when the mother is aged 35 years and over. Age affects the chances of having a Down syndrome child, a father aged 50 years has been shown to have an influence on the conception (conception) of a fetus with Down syndrome (Wiseman, 2015).

\section{Problems for People with Down Syndrome}

Children with this disorder require the same medical attention and treatment as normal children. They need health care, immunizations, medical emergencies, as well as support and guidance from their families. But there are several situations in which children with Down syndrome require special attention. Children with Down syndrome have reported hearing loss. Therefore it is necessary to have ear examinations from the beginning of life, as well as periodic hearing tests by an ENT specialist. 30-40\% of children with Down syndrome are accompanied by congenital heart disease. They require long-term treatment by a pediatric cardiologist (Katbamna \& Crumpton, 2011). Often experience visual disturbances or cataracts. So that it needs regular evaluation by an ophthalmologist. Some cases, especially those with other serious congenital abnormalities, will develop growth problems in infancy or preschool. Conversely, there are also cases of obesity in adolescence or after adulthood, so collaboration with nutritionists is needed.

Bone deformities can also occur in Down syndrome, which includes patellar dislocation, groin subluxation or atlantoacacial instability. If the latter causes depression of the spinal cord, or if the child holds the head in a torticollis-like position, then a radiological examination is required to examine the cervical spine and neurological consultation is required. Other medical aspects that require consultation with an expert include emunological problems, metabolic dysfunction or biochemical disorders.

Based on the description above, it can be concluded that the problems for people with Down syndrome are hearing impairment and impaired vision, congenital heart disease, growth problems during infancy / preschool, but there are also cases of obesity during adolescence, bone deformities such as bending. Down Syndrome Victoria, most children with Down syndrome experience several obstacles in all areas of development, including: Gross and fine motor skills Personal and social development, Language and speech development, and Cognitive development.

\section{Cognitive and behavioral problems}

The development of a child with Down Syndrome is certainly different from the development of a healthy child. Excessive expression of chromosomes leads to a decrease in the number of nerves in the central nervous system, delayed myelination, disruption of cell cycle regulation, and causes excessive protein production and abnormal neurotransmission. The existence of these conditions causes children with Down Syndrome to have problems with communication, concentration, memory, the ability to carry out tasks, motor development, and body control. 
Kim et al (2012), collected data on the motor and cognitive development profiles of children with Down Syndrome. Based on this study, children with Down's Syndrome have motor skills and require two times longer time to achieve developmental levels than normal children. Nearly all children with Down Syndrome have moderate intellectual impairment and unrelated cognitive and motor development. This is why children with Down Syndrome have difficulty focusing and concentrating.

The tendency for psychological and mental disorders in children with Down syndrome can cause compulsive obsession ( Markarian et al., 2010). Obsession is a thought or image that cannot be prevented and continues to exist in a person's consciousness even if he views it as something unpleasant and wants to avoid. A compulsion is a stereotypical act that encourages a person to repeat the action again and again even if he doesn't want to do it.

Due to their mental condition, Down syndrome children often display their unbalanced personality. Sometimes calm, sometimes chaotic, often pensive silence, but sometimes show tantrums (sulk), angry, irritable, annoy others, or make chaos and even damage.

\section{Conclusion}

Down syndrome is a condition of underdeveloped physical and mental development of children due to abnormalities in chromosomal development. As a result of physical and mental retardation, there are problems for people with Down syndrome, namely hearing impairments and impaired vision, congenital heart disease, growth problems during infancy / preschool. Not only health problems, people with Down syndrome also have cognitive and behavioral problems such as difficulty focusing, concentrating and carrying out tasks, compulsive and emotional behavior.

\section{References}

Abbasi Moheb, L. (2012). Identification of three novel genes for autosomal recessive intellectual disability and molecular characterization of the causative defects (Doctoral dissertation).

Antonarakis, S. E., Lyle, R., Dermitzakis, E. T., Reymond, A., \& Deutsch, S. (2004). Chromosome 21 and down syndrome: from genomics to pathophysiology. Nature reviews genetics, 5(10), 725-738

Fang, K. (2019). ALL THE LONELY PEOPLE: THE RELATIONSHIP OF LONELINESS AND SOCIAL ISOLATION TO AGE-RELATED CHANGES IN BRAIN STRUCTURE (Doctoral dissertation, Yale University).

Fisch, H., Hyun, G., Golden, R., Hensle, T. W., Olsson, C. A., \& Liberson, G. L. (2003). The influence of paternal age on Down syndrome. The Journal of urology, 169(6), 22752278.

Fisch, H., \& Braun, S. (2005). The male biological clock: The startling news about aging, sexuality, and fertility in men. Simon and Schuster.

Hulten, M., Patel, S., Jonasson, J., \& Iwarsson, E. (2009). On the origin of the maternal age effect in trisomy 21 Down syndrome: the Oocyte Mosaicism Selection (OMS) model. Reproduction.

Iverson, J. M., \& Braddock, B. A. (2011). Gesture and motor skill in relation to language in children with language impairment. Journal of Speech, Language, and Hearing Research. 
Katbamna, B., \& Crumpton, T. (2011). Impact of Neurodevelopmental Disorders on Hearing in Children and Adolescents. In Neurodevelopmental Disabilities (pp. 297-320). Springer, Dordrecht.Reviews Neuroscience, 16(9), 564-574.

Lautarescu, B. A., Holland, A. J., \& Zaman, S. H. (2017). The early presentation of dementia in people with Down syndrome: a systematic review of longitudinal studies. Neuropsychology review, 27(1), 31-45.

Markarian, Y., Larson, M. J., Aldea, M. A., Baldwin, S. A., Good, D., Berkeljon, A., ... \& McKay, D. (2010). Multiple pathways to functional impairment in obsessivecompulsive disorder. Clinical psychology review, 30(1), 78-88..

Pizzutillo, P. D., \& Herman, M. J. (2005). Cervical spine issues in Down syndrome. Journal of Pediatric Orthopaedics, 25(2), 253-259

Selikowitz, M. (2008). Down syndrome. The Facts.

Sullivan, K. A. (2013). The Early Start Denver Model: Outcomes and moderators of an intervention for toddlers with Autism (Doctoral dissertation).

Wiseman, F. K., Al-Janabi, T., Hardy, J., Karmiloff-Smith, A., Nizetic, D., Tybulewicz, V. L., ... \& Strydom, A. (2015). A genetic cause of Alzheimer disease: mechanistic insights from Down syndrome. Nature

Zaganjor, I., Sekkarie, A., Tsang, B. L., Williams, J., Razzaghi, H., Mulinare, J., ... \& Rosenthal, J. (2016). Describing the prevalence of neural tube defects worldwide: a systematic literature review. PloS one, 11(4), e0151586. 